\title{
Review on Performance of Static Random Access Memory (SRAM)
}

\author{
Santhiya. $\mathbf{V}^{1}$, Mathan. $\mathbf{N}^{2}$ \\ M.Tech, VLSI Design, Department of ECE, Sathyabama University, Chennai, India ${ }^{1}$ \\ Assistant professor, Department of ECE, Sathyabama University, Chennai, India ${ }^{2}$
}

\begin{abstract}
The power consumption is a major concern these days for long operational life. Although various types of techniques to reduce the power dissipation has been developed. One of the most adopted method is to lower the supply voltage. Techniques based on replica circuits which minimize the effect of operating conditions variability on the speed and power. In this paper different static random access memory are designed in order to satisfy low power, high performance circuit and the extensive survey on features of various static random access memory(SRAM) designs were reported.
\end{abstract}

Keywords: SRAM, Delay, speed and area, stability, Power Consumption.

\section{INTRODUCTION}

Random-access memory is a form of computer data computers. Dynamic RAM are considered volatile, as it storage. A random-access memory (RAM) device allows lost the information or data when power is removed from data items to be read and written in roughly the same the system.

amount of time regardless of the order in which data items are accessed[3]. In contrast, with some of the other direct access data storage media such as hard disks, DVD-RWs and CD-RWs, the time required to read and write data items varies significantly depending on their physical locations, due to mechanical limitations such as media rotation speeds and arm movement delays today, randomaccess memory (RAM) takes the form of integrated circuits (IC). Random Access Memory is normally associated with volatile type of memory such as DRAM memory modules, In these stored information is lost if the power is removed, many efforts have been made to develope non-volatile Random Access Memory chips[3]. Other types of non-volatile memory exist that allow random access for read operations, but it do not allow write operations or have limitations on them. These include most type of ROM and type of flash memory called NOR-Flash. The two main forms of modern RAM are

i) Static RAM (SRAM)

ii) Dynamic RAM (DRAM)

A. Static RAM: SRAM is a type of semiconductor memory that uses bistable latching circuitry to store each bit. The term static RAM differentiates it from dynamic RAM which must be periodically refreshed. Static RAM exhibits data remanence but it is still volatile in the conventional sense that data is eventually lost when the memory is not powered.

B. Dynamic RAM: DRAM stores a bit of data using a transistor and capasitor pair, which together comprise a Dynamic RAM memory cell. The capacitor holds a high/low charge, and the transistor acts as a switch that lets the control circuitry on the chip read the capacitor's state of charge or change it. This form of memory is less expensive to produce than static RAM, eventually it is the predominant form of computer memory used in modern

\section{II.SRAM OPERATION}

The SRAM cell has three different states such as standby, reading and writing. To operate in read mode and write mode SRAM should have "readability and "write stability" respectively.

A. Standby: If the word line is not asserted, the access transistor $\mathrm{m} 5$ and $\mathrm{m} 6$ disconnected the cell from the bit line[9]. The two cross coupled inverters formed and it will continue to reinforce each other as long as they are connected to the supply.

B. Reading: Let as consider 6T SRAM cell shown in figure.1. Assume that the content of the memory is 1, stored at Q. The read cycle is started by precharging both the bit lines to a logic 1 , then asserting the word line (WL), enabling both the access transistors $\mathrm{m} 5$ and $\mathrm{m} 6$ and then the second step occurs when the values stored in $\mathrm{Q}$ and Qbar are transferred to the bit lines by leaving BL at its precharged value and discharging $\mathrm{BL}$ through $\mathrm{m} 1$ and m5 to a logic 0 .

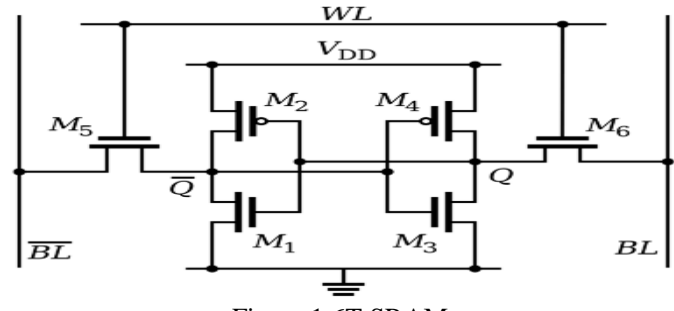

Figure.1 $\overline{6 \mathrm{~T}}$ SRAM

On the bit line (BL) side, the transistors $\mathrm{m} 4$ and $\mathrm{m} 6$ pull the bit line towards $V_{d d}$ a logical 1 . If the content of the memory was 0 , the opposite would happen and BL would be pulled toward 1 and BL toward 0[9]. Then the BL and BLbar line will have a small voltage differance between them while reaching sense amplifier[9]. It will sense which line has the higher voltage and then determining whether there was 1 stored or 0 . The higher the sensitivity of the sense amplifier and the faster the speed of the read operation. 
C. Writing: The start of a write cycle begins by applying the value to be written to the bit lines. If we wish to write 0 we would apply 0 to the bit line and 1 is written by inverting the values of the bit lines[11]. Word line (WL) is then asserted and the value that is to be stored is latched in. Bit line input drivers are designed to be much stronger than the relatively weak transistor in the cell, so that they can easily override the previous state of the cross coupled inverters.

D. Clock rate and power: The power consumption of SRAM varies widely depending on how frequently it is accessed, it can be as power hungry as dynamic random access memory (DRAM), when used at high frequency and some ICs can consume watts at full bandwidth[8]. On the other hand,static RAM used at a slower pace, such as in applications with moderately clocked microprocessors draws very less power and can have a nearly negligible power consumption when sitting idle in the region of a few micro watts.

E. Embedded use of SRAM: Static RAM in its dual ported form is used for realtime digital signal processing circuits[4]. Many categories of industrial and scientific subsystem, automotive electronics contain static random access memory. Several megabytes may be used in complex products such as digital cameras, cell phones, synthesizers etc. Static random access memory is also used in personal computers, routers, hard disk buffer etc[8].LCD screens and printers also normally employ static RAM to hold the image display.

\section{III.SURVEYED DESIGNS}

Shilpi Birla1 et. al (2011) analysed 8T Static Random Access Memory cell at $65 \mathrm{~nm}$ process technology is shown in figure.2. This topology was originally proposed for a subthershold static RAM design and optimized for functionality and performance over a large voltage rang. A write operation is performed through WWL, WBL and WBLX port, where as single ended read operation is exercised through RWL and RBL ports.RBL is precharged at the end of each read cycle and keep precharged during a write cycle. In this bit cell write and read ports are decoupled in contrast to the traditional 6T cell. Read-SNM problem is eliminated and 6T static RAM part can be sized for better writeablity without trading off RSNM. This makes the voltage drop across unaccessed read buffers zero and hence leakage on read bit line is highly reduced.

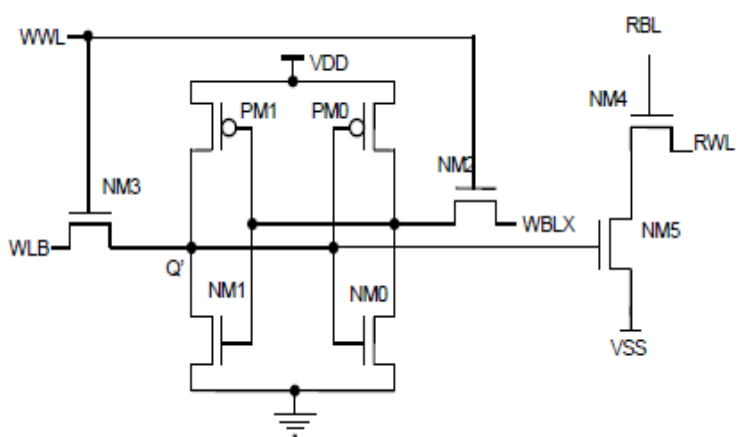

$\mathrm{V}_{\mathrm{dd}}$ is the virtual supply nodes for the cross coupled inverters and its voltage can be brought down during a write access to weaken PMOS load device and ease writeability problem at low voltage.Since all the bit cells on a row are written and read at the same time, $\mathrm{V}_{\mathrm{dd}}$ is shared across one row of memory cells.

J.Suganthi et. al (2012) proposed the design as shown in the figure. 3 operates fast as logic circuits operate and consumes a very less power at standby mode array. Nanometer SRAM cannot achieve lower $V_{\text {ddmin }}$ due to read disturb, half select disturb and write failure. Quantitative performance advantage of a zigzag 8T SRAM cell over the decoupled single ended sensing 8T-SRAM with write back schemes, as the most area efficient cell under large supply voltage variations.

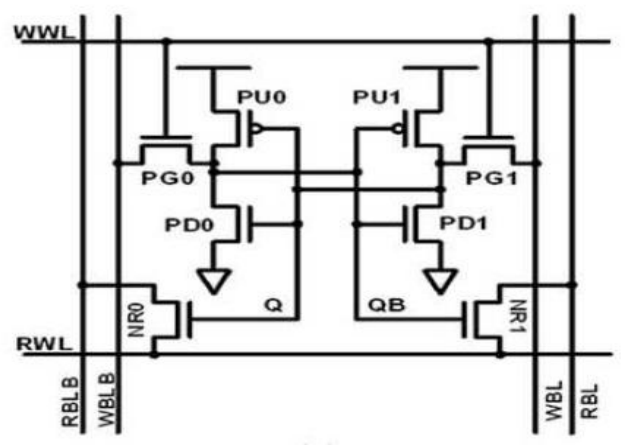

Figure.3 Zigzag 8T SRAM cell

Two decoupled read port transistors NR0 and NR1, are used to transfer the storage data $Q$ and $Q_{B}$ to the read bit line.The decoupled differential read port solves the read disturb issue. The zigzag 8T SRAM cell has better read static noise margins to the equivalent of hold SNM of conventional 6T static RAM.The zigzag 8T SRAM also has a faster read speed for long bit line application due to its small swing differential read sensing.

Armin Tajalli and Yusuf Leblebici (2009) proposed subthershold SCL(source coupled logic) for low power SRAM and low activity rate digital systems. The subthershold leakage consumption of conventional CMOS circuits is more pronounced subthershold SCL can be used effectively for reducing the power consumption. A 9T memory cell has been developed to reduce the stand-by current while the SRAM array is operating at $2.1 \mathrm{MHz}$ clock frequency. Proposed 9T memory cell is shown figure 4.

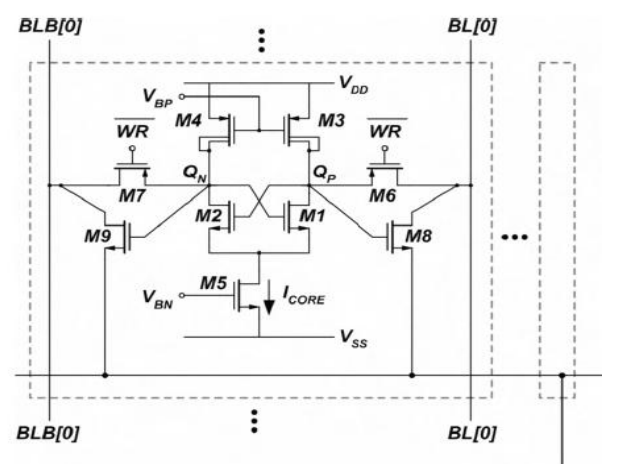

Figure. 4 9T SRAM cell 
The power consumption of the proposed circuit style is maintained in nanometer CMOS technology nodes.

Mr. Sunil Jadav et. al (2012) analysed ultra low power 6T SRAM using adiabatic technique is shown in figure 5. Adiabatic technique is used for reduction of average power dissipation.

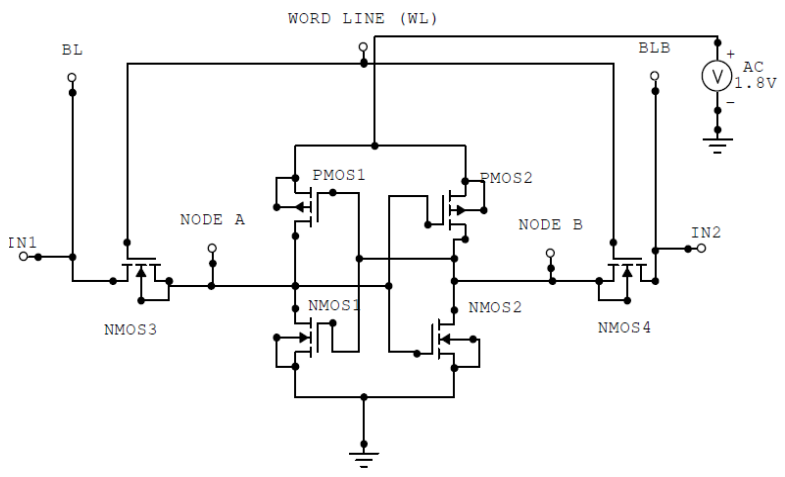

Figure $.56 \mathrm{~T}$ proposed SRAM cell

In this adiabatic technique ac power supply is used.By using the ac power supply rather than dc the average power dissipation is reduced. Adiabatic switching can be achieved by ensuring that the potential across the switching devices is kept aribitrarily small. Adiabatic charging may be achieved by charging the capacitor from time varying source that starts at $\mathrm{V}_{\mathrm{I}}=0 \mathrm{~V}$ to $\mathrm{V}_{\mathrm{dd}}$.

A.Priadarshini (2013) proposed low power CMOS Static Random Access Memory based field programmable gate arrays architecture and analysed CMOS SRAMs that are used for on chipe reconfiguration. This architecture is based on CMOS logic. Fast and low power SRAM based on 10T SRAM cells is shown in the figure 6 .

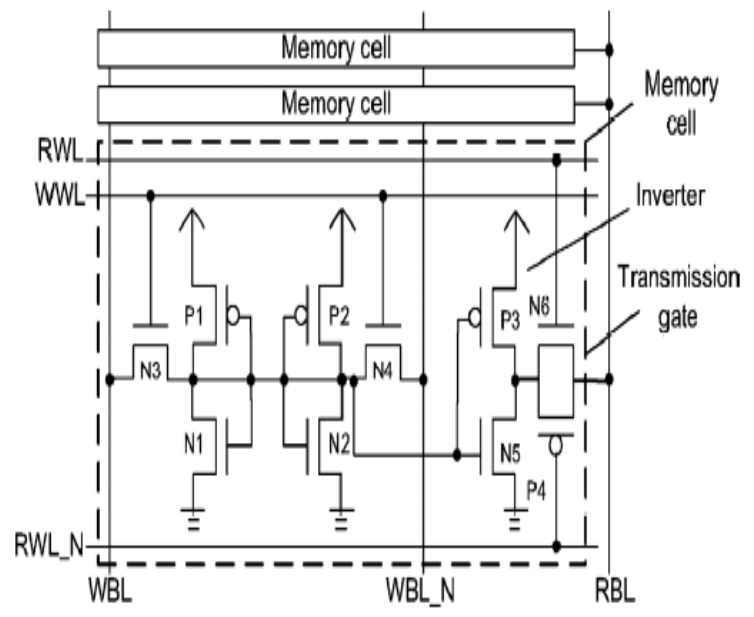

Figure. 6 10T SRAM cell

Compared to conventional 4T and 6T SRAM cells delay is improved and the time for precharged is reduced, 10T SRAM design avoids high switching activities on memory read bitlines and thus saves most of the charge or precharge power.

Meenatchi Jagasivamani and Dong Sam Ha (2001) analysed low power compiler with the capability to choose between a low power and high speed SRAM. Structure of SRAM compiler is shown in figure. 7.

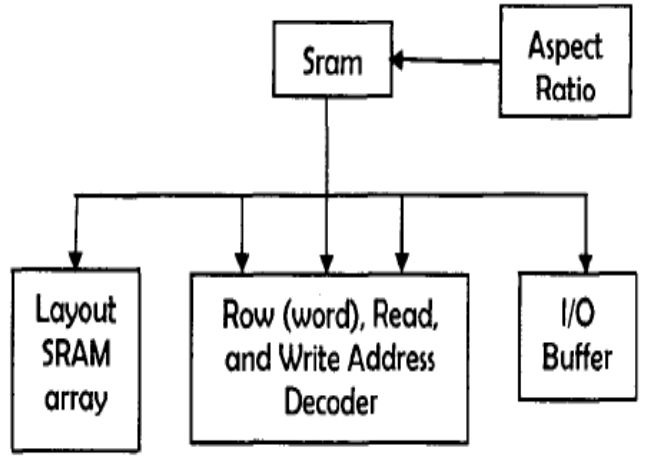

Figure 7 Structure of SRAM compiler

Overall average power dissipation is reduced and substantial power over non-partitioned SRAM at the cost of speed and area.

Ankita Singh (2014) analysed the design of 8T SRAM using energy recovery logic and by using the energy recovery logic cell stability, leakage noise has been improved. Figure 8 shows the 8T SRAM using energy recovery logic.

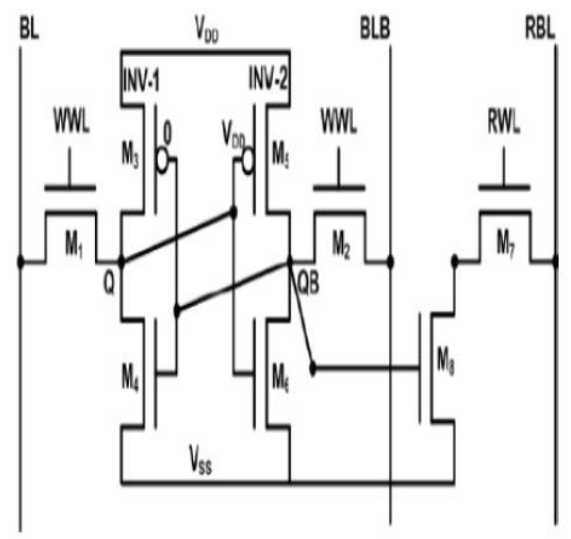

Figure.8 8 T SRAM using energy recovery logic

The proposed SRAM is structurally close to the conventional CMOS SRAM. The PMOS transistor connected between power line and $\mathrm{V}_{\mathrm{dd}}$ and NMOS is connected to GND and $\mathrm{V}_{\mathrm{dd}}$. Energy recovery signal is input from bit line. While the input voltage increases, the other voltage depends on the circuit.

\section{CONCLUSION}

An extensive survey has been done for various design of Static Random Access Memory. These designs are well preferred for various low power applications. Various techniques to reduce the power dissipation has been developed and it can be used for low power and high speed applications.

\section{REFERENCES}

[1] Akamatsu.H, Satomi.K, Suzuki.T, Yamagami.Y and Yamauchi.H,“A stable 2-port SRAM cell design against simultaneously read/write disturbed accesses" IEEE J. Solid-State Circuits, vol. 43, no. 9, pp.2109-2119,2008.

[2] Ankita Singh,"Analysis of 8T SRAM using Energy Recovery Logic", IJERT, vol.3 Issue 2, feb 2014. 
[3] Anurag Dandotiya and Amit S. Rajput," SNM Analysis of 6T SRAM at 32NM and 45NM Technique," IJCA, vol.98, no.7, July 2014.

[4] Armin Tajalli and Yusuf Leblebici, "Subthreshold SCL for Ultra-LawPower SRAM and Low-Activity-Rate Digital Systems,"

[5] J. Caravella, "A Low Voltage SRAM For Embedded Applications," IEEE Jourrial of Solid-state Circuits, Vol. 32, No. 3, pp. 428- 432, Mar. 1997.

[6] V. Gupta, M. Anis, "Statistical design of the 6T SRAM bit cell", IEEE Trans. Circuits Syst. I, Vol. 57, No. 1, pp. 93-104, Mar. 2010.

[7] Meenatchi Jagasivamani and Dong Sam Ha, "Development of Low Power Compiler", 2001.

[8] Nahid Rahman and B. P. Singh, "Design and Verification of Low Power SRAM using 8T SRAM Cell Approach," IJCA, Vol. 67, no.18, April 2013

[9] Naveen Verma,"'Ultra-Low-Power SRAM Design In High Variability Advanced CMOS," May 2009.

[10] A.Priadarshini, "Low power reconfigurable FPGA based on SRAM," ICCCI , Jan 2013.

[11] Saurabh Neemgaonkar, Anshul Jain, Vijay Kumar Magraiya," Comparative Analysis of Low Power 8T SRAM," IJECT, vol.4, Issue-4, 2013.

[12] Mr. Sunil Jadav, Mr. Vikrant, Dr. Munish Vashisath, "Design and Performance Analysis of Ultra Low Power 6T SRAM Using Adiabatic Technique," IJVLSICS, Vol.3, No.3, June 2012.

[13] N.Mathan, "CNTFET based Highly Durable Radix-4 Multiplier using an Efficient Hybrid Adder", Biosciences Biotechnology Research Asia, December 2014, Vol. 11(3), pp 1855-1860

[14] Shilpi Birla1, Neeraj Kumar Shukla, Kapil Rathi, Rakesh Kumar Singh, Manisha Pattanaik,"Analysis of 8T SRAM Cell at Various Process Corners at $65 \mathrm{~nm}$ Process Technology," Circuits and Systems, pp.326329, 2011.

[15] J.Suganthi, N.Kumaresan, K.Anbarasi, "Design of Low Power Zigzag 8T SRAM array with Differential Write Back Scheme," IJEAT, Vol-1, Issue-6, June 2012.

[16] N. Verma and A. P. Chandrakasan, "A $256 \mathrm{~kb} 65 \mathrm{~nm}$ 8T subthreshold SRAM employing sense-amplifier redundancy" IEEE J. oj Solid-State Circuits, vol. 43, no. I, pp. 141-149, Jan. 2008.

\section{BIOGRAPHIES}

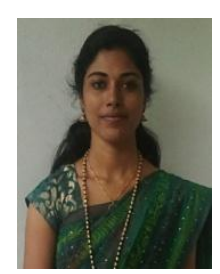

Santhiya.V was born in Nagercoil, Tamil Nadu, India in 1992. She received his Bachelor Degree in Electronics and communication from Anna University, Chennai in the year of 2013. She is pursuing her Master Degree in VLSI Design in Sathyabama University. Her interested areas are VLSI design and Signal Processing.

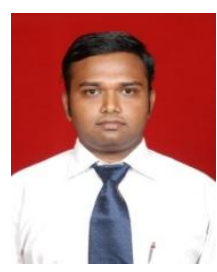

Mathan.N was born in Nagercoil, Tamilnadu, India in 1989. He received his Bachelor Degree in Electronics and Communication Engineering from Anna University, Chennai in the year 2010. Master Degree in VLSI Design from Sathyabama University, Chennai in the year 2013. He is working as Assistant Professor in Department of ECE in Sathyabama University. His interested areas are Nano Electronics, VLSI Design, Low Power VLSI Design, Testing of VLSI circuits, Wireless Sensor Networks and Advanced Digital System Design. He has Research publications in National / International Journals /Conferences 